\title{
The detection of light scattered from stimuli in impaired regions of the visual field
}

\author{
MALCOLM E. WILSON ${ }^{1}$
}

From the Institute of Neurology, Queen Square, London

\begin{abstract}
'What is the most intense stimulus I should use when studying an impaired region of the visual field?' This is a question which frequently faces the clinical investigator who is studying the function of a severely impaired region of the visual field, but it is a question to which answers cannot be found in the literature.
\end{abstract}

The question arises because there are two distinct ways in which an intense stimulus, directed into a defective region of the field, may be detected. First, the stimulus may be seen simply because it lies above the threshold for the defective region. On the other hand, it is known that any light passing through the intraocular media suffers a small degree of scattering and illuminates a wide area of retina. If a stimulus is unusually intense, this scattered light may be detected by unimpaired regions of the field, even though the stimulus itself is still below threshold for the defective region in which it lies.

In studying the function of an impaired region of the field one must, therefore, be sure that the patient is not detecting the light scattered from the stimulus. The only way to ensure this, is to keep the stimulus intensity below that at which scattered light is potentially detectable - that is, below the scattered-light threshold for the defective region under consideration.

A number of studies of various aspects of intraocular light scatter have been reported (Stiles, 1928; Brindley and Willmer, 1952; Boynton and Clarke, 1962; Wolf and Morandi, 1962). Other authors have noted artefacts resulting from intraocularly scattered light-for example, Boynton and Riggs (1951), and Hubel and Wiesel (1960). Unfortunately, none of these authors were primarily concerned with the parameters determining the visibility of scattered light, and their data do not provide the scattered-light thresholds which the clinical investigator needs.

It is the purpose of this paper to present, in a

1 Present address: Neurophysiology-Biophysics Research Unit, Veterans Administration Hospital, Boston, Mass., 02130, U.S.A. clinically useful form, some direct measurements of the scattered-light thresholds of 'blind' regions of the field.

\section{METHODS}

EXPERIMENT I: STIMULI IN THE PHYSIOLOGICAL BLIND SPOT Apparatus Stimuli were presented at the centre of a uniform white field of luminance $200 \mathrm{~cd} / \mathrm{m}^{2}$. This field was provided by the concave surface of a hemisphere $1 \mathrm{~m}$ in radius. The subject was seated facing into the hemisphere. His head was positioned so that the eye to be tested was located at the centre of the open side of the hemisphere.

Stimuli were seen in Maxwellian view through a variable aperture in the centre of the hemisphere surface. The diameter of each stimulus was limited by this aperture, and its duration was controlled by an electro-mechanical shutter. Five different stimuli were employed. Their diameters were $8 \cdot 8,14 \cdot 1,28 \cdot 3,46 \cdot 8$, and $80.8 \mathrm{~min}$ arc, and all had a duration of $1 \mathrm{sec}$. The stimulus aperture was continually lighted by a second Maxwellian view system so that its brightness, between stimulus presentations, would match that of the surrounding hemisphere. Only white light from incandescent tungsten sources was employed in this experiment.

The position of the stimulus in the visual field was determined by the position of a movable fixation mark on the hemisphere surface. The subject's fixation on this mark was monitored by the experimenter by means of a telescope which passed through the wall of the hemisphere. Eye movements of more than $1^{\circ}$ were readily observable. Rigid head fixation was not necessary in this experiment, because the images of the light sources for the two Maxwellian view optical systems exceeded $2 \cdot 2 \mathrm{~cm}$ in diameter when focused at the subject's pupil. Head movements large enough to place the pupil outside these images were readily seen through the telescope used to monitor fixation. The natural pupil was used throughout the experiment.

Procedure Three hospital patients served as subjects. Their central visual fields were first charted on a tangent screen to ensure that the region surrounding the physiological blind spot was normally sensitive.

The patient was then seated at the hemisphere, and the position of the fixation point which would place the stimuli in the centre of his blind spot was determined. 
The stimuli, therefore, lay $2-3^{\circ}$ from a normally sensitive part of the field. The threshold luminances of five stimuli of differing diameter were then measured. The order of the threshold determinations was different in each of the patients.

The patient was required to make repeated choices between a presentation of the stimulus itself, and a 'blank'. He heard two auditory tones, $1 \mathrm{sec}$ apart, and during one of these tones the stimulus was presented. The patient was then asked whether the stimulus had coincided with the first or with the second tone. A forcedchoice procedure of this kind is necessary when naive observers are faced with a task as unfamiliar as the detection of scattered light. If they are simply asked whether they saw the stimulus, they may well fail to report the transient overall glare which is produced by stimuli in blind regions of the field.

The threshold was defined as the luminance at which the patient correctly distinguished between the stimulus and the 'blank' on $80 \%$ of the presentations. The sequence of stimulus luminances used in locating the threshold followed a modified method of adjustment. This procedure has been described and validated elsewhere (Wilson, 1965), and will not be described in this paper.

EXPERIMENT II: STIMULI IN PERIMETRICALLY 'BLIND' REGIONS OF THE VISUAL FIELD Apparatus The apparatus was essentially the same as in Experiment $I$, but two features were added to make it possible to change the hemisphere luminance in the area immediately surrounding the stimuli. To decrease the luminance, the stimulus aperture was surrounded by a black disc, $15^{\circ}$ in radius. The luminance of this disc was $1.23 \mathrm{log}$ units below that of the remainder of the hemisphere. To increase the hemisphere luminance around the stimulus, a patch of light was projected onto the hemisphere so that the stimulus lay at its centre. The luminance of this square of light was $1.0 \mathrm{log}$ units above that of the remainder of the hemisphere. Whenever the stimulus surround was altered in luminance, the luminance of the stimulus aperture was adjusted to match it. The three alternative stimulus backgrounds - the disc, the unaltered hemisphere, and the square-will be referred to as, respectively, the dim, the standard, and the bright stimulus backgrounds.

Procedure Four patients took part in this experiment. All had visual field defects as a consequence of damage involving the optic radiation, or the striate cortex, or both.

\section{Patients}

1. Male, aged 32 years. Dense right homonymous hemianopia of six months' duration. The defect was of sudden onset and was preceded by a five-year history of occasional attacks of 'migraine' with visual disturbances in the right visual field. The patient showed no other neurological abnormality.

A vertebral arteriogram showed very poor filling of one of the occipital branches of the left posterior cerebral artery, compared with the corresponding branch on the right. The patient was diagnosed as having a vascular lesion in the left striate cortex.
2. Female, aged 38 years. Dense homonymous defect in the left upper quadrant, with a less dense, and onlyD partially congruent, homonomous defect in the left lowe quadrant.

The patient was tested one month after partial remova $\overline{\bar{C}}$ of an astrocytoma involving the right temporal lobe. The neoplasm extended medially and also into the righe? parietal region.

3. Female, aged 50 years. Incomplete right homony mous hemianopia, densest in the lower quadrants. The defect was of sudden onset and had been present for three weeks. No other neurological abnormalities weres? found. The patient was presumed to have a vascularo lesion involving the left optic radiation, striate cortex, o both.

4. Male, aged 58 years. Incomplete left homonymousD hemianopia, dense in the upper quadrant of the field, les dense in the lower quadrant. The defect was of suddens onset and had been present for two and a half months $\overrightarrow{0}$ No other neurological abnormality was found and the patient was presumed to have a vascular lesion involving $\overrightarrow{\omega_{j}}$ the right optic radiation, striate cortex, or both.

The visual fields of these patients were determined by the usual perimetric methods and they are presented in fult elsewhere (Wilson, 1965: subjects $\mathrm{H} 2,3,4$, and 6). In every case the defect was confined to one half of the visual field. In at least one quadrant of the impairedr field, the defect was so dense that a white card, $10^{\circ}$ squ年e and moving against a black background, was undeteote able. Such parts of the field were regarded as pesi metrically 'blind'.

The luminance required for the detection of a stimuKuss projected into a perimetrically 'blind' field was therp determined in each patient. The stimuli lay between and $30^{\circ}$ from the fixation point, but their exact locationwas different in each of the four subjects. The standats stimulus background was used during these threshold ${ }^{\circ}$ determinations.

Although the regions of the field in which the stimuli lay, were perimetrically 'blind', it cannot be assumede that the direct retino-cortical pathways from that region of the field were totally non-functional. The possibility remains that the unusually intense stimuli employed in measuring the detection threshold were able to activat $\vec{\xi}$ fibres unresponsive to less intensive stimulation. This possibility must be excluded if the thresholds are to be regarded as due to light scattered intraocularly. The effect of changing the luminance of the background on which the stimuli were presented was, therefore, investigated It was reasoned that if the stimuli were detected as ap result of activity in the damaged retino-cortical nerve fibres, then local changes in the stimulus background. should alter the stimulus threshold; much as they do if normal visual fields. In contrast, if the stimuli were being detected as a result of activity in unimpaired fibres remotes from the stimulus, the stimulus threshold should be relatively independent of changes in its background.

The effect of dimming the background was measuredE. in only one patient, in view of the report of Krieger and Bender (1949), that adaptation to dim backgrounds is slow and incomplete in impaired visual fields. In the three remaining patients the effect of increasing the्W 
background luminance was determined. In all four patients the stimulus was presented in a densely impaired region of the field and its threshold determined first on the standard background and then on the dim (or the bright) background. For purposes of comparison, the two threshold determinations were repeated at the same distance from the fixation point, but in a normal part of the field.

\section{RESULTS}

EXPERIMENT I: STIMULI IN THE PHYSIOLOGICAL BLIND SPOT Figure 1 is plotted to show the threshold luminance of a stimulus as ordinate, and its area as abscissa. Both axes are logarithmic.

The points on the line show the threshold luminance for each of the five different stimuli which were presented in the physiological blind spot. Each point was derived by arithmetically averaging the thresholds determined in the three patients. The vertical line through each point represents the standard deviation of the three threshold determinations.

How is the scattered-light threshold of the blind spot to be expressed? It is apparent from the line in Fig 1, that the threshold luminance of stimuli in the blind spot varies widely, becoming lower as larger stimuli are used. However, if the sum of

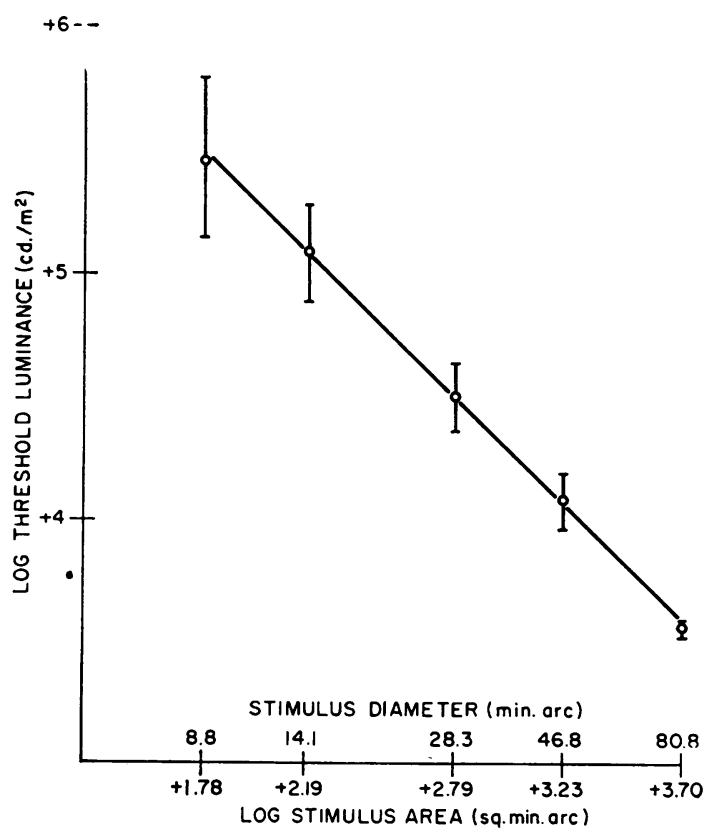

FIG. 1. Thresholds for the detection of light scattered from stimuli presented in the physiological blind spot. Background luminance $200 \mathrm{~cd} / \mathrm{m}^{2}$. (58 ft. L). the $\log$ stimulus luminance and the log stimulus area (a measure of stimulus flux) is calculated for the points on the line, it is found to be the same for stimuli of any area. For example, for a stimulus $8 \cdot 8^{\prime}$ in diameter this sum is $+\mathbf{7 \cdot 2 5}$. For a stimulus $80.8^{\prime}$ in diameter the sum is $+7 \cdot 26$. Therefore, we may say that a stimulus in the blind spot was detected when the sum of its log luminance (in $\mathrm{cd} / \mathrm{m}^{2}$ ) and its $\log$ area (in sq. $\min$ arc) reached $\mathrm{a}$ mean value of $+7 \cdot 25$.

Now, if an experimenter is to be reasonably confident $(P>0.998)$ that the light scattered from a stimulus in the blind spot will not be detected, then the stimulus flux should lie at least threestandard deviations below the mean threshold value. The greatest standard deviation found in the threshold flux determinations in the blind spot was $0.35 \mathrm{log}$ units (see Fig. 1). Therefore, if the light scattered from a stimulus in the blind spot is to be undetectable, the sum of the log stimulus luminance (in $\mathrm{cd} / \mathrm{m}^{2}$ ) and its $\log$ area (in sq. min arc) should not exceed $+7 \cdot 25-1.05=+6.20$. A simpler and more generally useful form of this expression will be developed in the Discussion.

EXPERIMENT II : STIMULI IN DENSELY IMPAIRED REGIONS OF THE FIELD The threshold luminances of stimuli presented in densely impaired parts of the field are shown on the first line of Table I. The diameters and $\log$ areas of these stimuli are shown on the second and third lines. The sum of the log threshold luminance and the log area of each stimulus is shown on the fourth line. The mean value of this sum for the four patients was +8.34 ; a value 1.09 log units higher than that found for stimuli

\section{TABLE I}

THRESHOLDS OF STIMULI PRESENTED IN DENSELY IMPAIRED REGIONS OF THE VISUAL FIELD

Patient

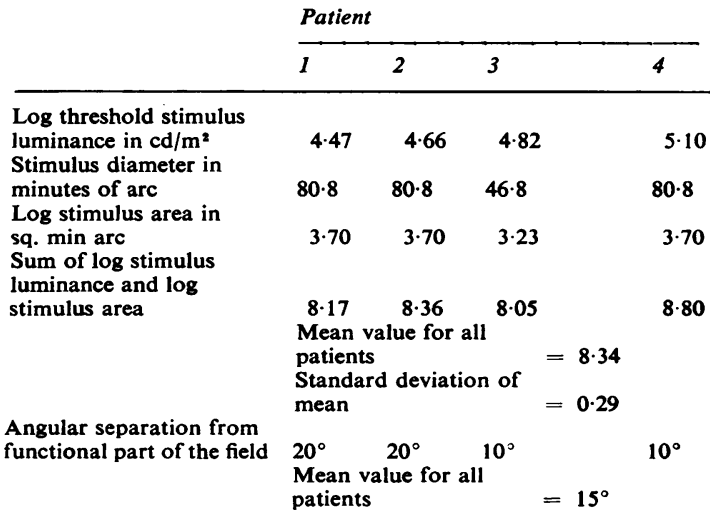


of the same diameter presented in the physiological blind spot.

The angles which separated the stimuli in the densely impaired parts of the field from the nearest functional part of the field are shown on the lowest line of Table I. (A region of the field was regarded as 'functional' if the patient could detect a moving, white, tangent-screen target smaller than 40/2000 $\left(1.2^{\circ}\right.$ in diameter) within it.) These angles ranged from $10^{\circ}$ to $20^{\circ}$ arc, with a mean value of $15^{\circ}$ for the four patients.

The mean threshold value ' +8.34 ' is a measure of the mean flux which a stimulus must have if it is to be detected when it lies $15^{\circ}$ into a 'blind' field. If such a stimulus is to be undetectable, then its flux should lie at least three standard deviations below this mean threshold value. Table I (fourth line) shows that the standard deviation of the threshold fluxes, measured at $15^{\circ}$ into a 'blind' part of the field, was $0.29 \mathrm{log}$ units. Therefore, if a stimulus $15^{\circ}$ into a 'blind' field is to be seen with a probability of less than 0.002 , the sum of its $\log$ luminance (in $\mathrm{cd} / \mathrm{m}^{2}$ ) andits $\log$ area (in sq. $\min$ arc) should not exceed $+8.34-0.87=+7.47$. This expression will be simplified in the Discussion.

In order to show that the detection of stimuli in densely impaired fields was mediated by activity in unimpaired visual fibres remote from the stimulus; the effect on these thresholds of changing the local stimulus background luminance was studied. The results are presented in Table II.

\section{TABLE II}

THESHOLD CHANGES IN IMPAIRED AND NORMAL REGIONS OF THE FIELD, RESULTING FROM CHANGES IN THE LUMINANCE OF THE STIMULUS BACKGROUND

\begin{tabular}{|c|c|c|c|}
\hline Patient & $\begin{array}{l}\text { Change in back- } \\
\text { ground luminance } \\
(\log u .)\end{array}$ & $\begin{array}{l}\text { Change in thres- } \\
\text { hold luminance in } \\
\text { normal field } \\
(\log u .)\end{array}$ & $\begin{array}{l}\text { Change in thres- } \\
\text { hold luminance in } \\
\text { impaired field } \\
(\log u .)\end{array}$ \\
\hline 1 & $\begin{array}{l}\text { Decrease of } 1.23 \\
\text { Increase of } 1.0 \\
\text { Increase of } 1.0 \\
\text { Increase of } 1.0\end{array}$ & $\begin{array}{l}73 \\
7 \\
9 \\
3\end{array}$ & $\begin{array}{r}0.09 \\
0.24 \\
0.05 \\
0.05\end{array}$ \\
\hline
\end{tabular}

In patient 1 , the background luminance was decreased by $1.23 \mathrm{log}$ units. When the stimulus lay in an unimpaired part of the field, this change in the background luminance substantially decreased the stimulus threshold luminance, as was expected. (The decrease was $0.73 \mathrm{log}$ units.) In contrast, when the stimulus lay in a densely impaired part of the field, the same change in the background luminance scarcely altered the stimulus threshold. (The change was an increase of only $0.09 \mathrm{log}$ units.) Therefore, we may conclude that the threshold determined in $\bar{Z}$ the densely impaired region of the field in this $\stackrel{\mathbb{Q}}{\square}$ patient was mediated by visual pathway fibres remote from the stimulus.

In patients 2,3 , and 4 , the background luminance 0 was increased by $1.0 \mathrm{log}$ units. When the stimuli ${ }_{W}$ lay in an unimpaired part of the field, this background change caused a substantial rise in the threshold luminance of the stimuli, as was expected. The mean increase for the three subjects was $0.93 \log$ units. In contrast, when the stimuli lay in densely $\underset{?}{\vec{S}}$ impaired parts of the field, the same increase in the $\frac{\sigma}{0}$ background luminance caused a mean stimulus $\frac{}{O}$ threshold increase of only $0.05 \log$ units. Therefore, $\overline{\frac{\omega}{7}}$ it may be concluded that the thresholds determined $\overparen{\nabla}$ in the impaired fields of patients 2-4 were also mediated by fibres subserving parts of the field remote from the stimulus.

These results establish that in all four patients the stimuli presented in perimetrically blind parts of the field were detected by excitation of nerve pathways subserving unimpaired parts of the field. They do not, however, show how the excitation $\omega$ reached these fibres, and the possibility remains ir that it was by way of lateral neural interconnectiong below the level of the lesion, rather than because of intraocularly scattered light.

Although this possibility cannot finally be discounted by the present experiments, there is 0 reason to think that the mechanism of excitatio $\overrightarrow{0}$ was in fact light scatter. When, in Experiment stimuli were presented in the physiological blin $\vec{\Phi} \cdot \vec{\theta}$ spot, subjects reported seeing the scattered light ast a surrounding 'glare', brighter near the margins of the blind spot than further away. A very similar gradient of 'glare,' brightest towards the edges of the 'blind' region, was reported on a number of occasions by subjects in Experiment II, even when the stimuli were close to threshold. This observation strongly supports the view that the thresholds determined in densely impaired fields were scatteredlight thresholds.

\section{DISCUSSION}

How intense may we make a stimulus in a deniely impaired field without running the risk that the patient will detect scattered light? The thresholds determined in the physiological blind spot provide the most conservative estimate of this limit. Stimuli in the blind spot, lying $2-3^{\circ}$ from normally sensitive field, were undetectable $(P>0.998)$ so long as the sum of their $\log$ luminance (in $\mathrm{cd} / \mathrm{m}^{2}$ ) and their $\log$ area (in sq. min arc) did not exceed $+6 \cdot 20$. It follows that the light scattered from a stimulus in a densely impaired field will also remain undetectable 
so long as the stimulus lies at least $2-3^{\circ}$ from normal field, and the sum of its log luminance and $\log$ area is less than $+6 \cdot 20$.

As it stands, this limiting value depends on the units in which the stimulus parameters are measured. Furthermore, it is applicable only to stimuli presented on backgrounds whose luminance is the same as that of the background used in these experiments $\left(200 \mathrm{~cd} / \mathrm{m}^{2}\right)$. However, it may readily be given a form which is both more convenient and more generally applicable.

First, the parameter 'log stimulus luminance' may be replaced by the ratio of the stimulus luminance to the background luminance $\left(\operatorname{Lum}_{\mathrm{S}} /\right.$ $\left.\operatorname{Lum}_{B}\right)$. This ratio is independent of the units in which luminance is measured. Further, Steinhardt (1936) showed that the threshold value of this ratio is largely independent of changes in the background luminance, provided that this luminance exceeds $75 \mathrm{~cd} / \mathrm{m}^{2}$ (22 ft. L.). Secondly, the parameter ' $l o g$ stimulus area' may more conveniently be replaced by the square of the stimulus diameter in degrees.

When the results are expressed in these terms, we find that the light scattered from a stimulus presented at least $2-3^{\circ}$ into a densely impaired field is unlikely to be detected so long as;

Lum $_{\mathbf{S}} / \operatorname{Lum}_{\mathbf{B}} \mathbf{X}$ (Stimulus diameter in degrees) ${ }^{2}<$ $2 \cdot 8$

This limit applies to stimuli whose duration is $1 \mathrm{sec}$, presented on backgrounds whose luminance is greater than $75 \mathrm{~cd} / \mathrm{m}^{2}$.

The value ' 2.8 ' is based on thresholds which were certainly mediated by scattered light, and this limit may be applied safely to any stimulus presented $3^{\circ}$ or more from functional visual field. However, the results of Experiment II suggest that when stimuli lie appreciably more than $3^{\circ}$ from functional visual field, their flux may be increased beyond this limit without risk that the patient will detect scattered light. When these results are expressed in terms of stimulus background contrast and stimulus diameter, it is found that the light scattered from a stimulus $15^{\circ}$ into a densely impaired region of the visual field is unlikely to be detected $(P<0.002)$ so long as;

Lum $_{S} / \operatorname{Lum}_{B} X$ (Stimulus diameter in degrees) $)^{2}<53$

If a stimulus were presented between $3^{\circ}$ and $15^{\circ}$ into an impaired part of the field, the limiting flux would probably have a value between $2 \cdot 8$ and 53 .

In conclusion, it must be emphasized that these limits are approximate. They may well be modified by such factors as the configuration of a particular visual field defect, and the psychophysical procedure used to determine whether the stimulus has been detected. However, they do provide a convenient indication of the conditions under which serious consideration must be given to the possibility that a patient is detecting the light scattered from a stimulus.

\section{SUMMARY}

Psychophysical thresholds for the detection of light scattered from stimuli lying in blind regions of the visual field have been measured. Stimuli presented $2^{\circ}-3^{\circ}$ arc from a functional part of the field are unlikely to be detected, provided that the product of the stimulus/background luminance contrast and the square of the stimulus diameter (in square degrees) does not exceed 2.8. Stimuli lying $15^{\circ}$ from a functional part of the field are unlikely to be detected so long as the value of this product is less than 53. Both limits apply to stimuli $1 \mathrm{sec}$ in duration, presented on backgrounds whose luminance exceeds $75 \mathrm{~cd} / \mathrm{m}^{2}$ (22 ft. L.).

I am grateful to the physicians and surgeons at the National Hospitals for Nervous Diseases for giving me access to patients in their care. The experimental work reported in this paper was supported by the Medical Research Council, and the subsequent analysis of data by the U.S. Veterans Administration.

\section{REFERENCES}

Boynton, R. M., and Clarke, F. J. J. (1962). Sources of entoptic scatter in the human eye. J. opththal. Soc. Amer., 52, 1326.

intensity upon the human retinal response. J. exp. Psychol., 42, 217-226.

Brindley, G. S., and Willmer, E. N. (1952). The reflexion of light from the macular and peripheral fundus oculi in man. $J$. Physiol. (Lond.), 116, 350-356.

Hubel, D. H., and Wiesel, T. N. (1960). Receptive fields of optic nerve fibres in the spider monkey. Ibid., 154, 572-580.

Krieger, H. P., and Bender, M. B. (1949). Dark adaptation in lesions of the optic pathways. Fed. Proc., 8, 89.

Steinhardt, J. (1936). Intensity discrimination in the human eye. J. gen. Physiol., 20, 185-209.

Stiles, W. M. (1928). The effect of glare on the brightness difference threshold. Proc, roy. Soc. B., 104, 322-351.

Wilson, M. A. (1965). Spatial and Temporal Summation in Defective Fields of Vision. Unpublished Ph.D. Thesis, University of London.

Wolf, E., and Morandi, A. J. (1962). Retinal sensitivity in the region of the blind spot. J. opththal. Soc. Amer., 52, 806-812. 